\title{
Structural and electronic changes of pentacene induced by potassium doping
}

\author{
A. Guijarro \\ Departamento de Química Orgánica and Instituto Universitario de Síntesis Orgánica, Universidad de Alicante, \\ San Vicente del Raspeig, 03690 Alicante, Spain \\ J. A. Vergés* \\ Departamento de Teoría y Simulación de Materiales, Instituto de Ciencia de Materiales de Madrid (CSIC), \\ Cantoblanco, 28049 Madrid, Spain
}

(Received 13 February 2017; published 24 April 2017)

\begin{abstract}
Potassium is introduced into the crystalline herringbone structure of pentacene searching for a compound showing metallic electronic transport properties and, hopefully, superconductivity at small enough temperatures. Several possible structures for stoichiometric KPentacene (1:1), $\mathrm{K}_{2}$ Pentacene (2:1), and $\mathrm{K}_{3}$ Pentacene (3:1) compounds are theoretically investigated. Detailed densities of states for all of them are presented. As a more prominent result, a new monoclinic structure has been stabilized for the potassium richer material that could correspond to the recently synthesized superconducting phase of $\mathrm{K}_{3}$ Pentacene. Although energetically unfavorable, it is the only metallic candidate found to date.
\end{abstract}

DOI: 10.1103/PhysRevB.95.134112

\section{INTRODUCTION}

Doping of molecular crystals formed by certain polycyclic aromatic hydrocarbon $(\mathrm{PAH})$ molecules like picene has originated a new class of superconducting materials of very promising characteristics. The pioneering work of Mitsuhashi et al. [1] showed superconductivity of potassium-intercalated picene at $7 \mathrm{~K}$ or $18 \mathrm{~K}$ depending on sample processing. Later, other PAH crystals showing a similar laminar structure in which planes are formed by molecules stacking in a herringbone structure have also shown superconducting properties according to the temperature behavior of magnetic susceptibility. Until now, there is only one report of direct measurement of zero resistivity in this area [2]. The review by Kubozono et al. provides a good starting point to learn about this emerging field [3].

Although parallel to the experimental work there have been many theoretical calculations of the electronic structure of potassium-intercalated herringbone structures, a definite consensus on the precise position of dopant potassium atoms has not been reached. Experimental samples are not crystalline enough to allow a precise structural determination using $\mathrm{X}$-ray diffraction. The lack of this information is particularly relevant when comparing the doping of picene crystals with the doping of pentacene crystalline samples. Both elongate molecules are formed by five benzene rings, picene showing armchair sides that contrast with the zigzag sides of pentacene. Both are stacked in a herringbone layered structure in the pristine crystal. Potassium enters within the PAH herringbone structure (intralayer doping) where there is space enough for three dopant atoms. This was the consensus immediately after the discovery of superconducting properties of K-doped picene [1,4-9].

On the other hand, it was widely assumed that doping pentacene with alkaline metals only improves conductivity for less than one dopant per pentacene molecule. Potassium,

\footnotetext{
*jav@icmm.csic.es
}

in particular, would occupy interlayer positions donating one electron to pentacene semiconducting bands. In this way, n-type semiconducting behavior could be understood [10]. Some years later, experiments carried out by Mori and Ikehata on potassium doped pentacene showed an unusual kind of magnetic transition at low temperatures [11]. Potassium uptake was near to the 3:1 ratio, but location of potassium atoms was not further investigated [12]. Quite a few years later, Hansson et al. conducted the first numerical research on the atomic and electronic structures of potassium-doped pentacene [13]. Again small doping (KPentacene ${ }_{2}$ ) would correspond to $\mathrm{K}$ interlayer positions but larger doping (KPentacene) would completely modify the herringbone structure of pristine pentacene: $\mathrm{K}$ would penetrate within the $\mathrm{PAH}$ region mutating the layer structure of the original crystal. The electronic band structures calculated for both compounds show relatively broad bands that point to a conventional metallic behavior. Unfortunately, subsequent experimental work contradicted this explanation: Potassium-intercalated pentacene shows metallic behavior in a broad range of $\mathrm{K}$ concentrations (less than one atom per pentacene molecule) but not for KPentacene [14]. Again, the authors of this work supposed that potassium atoms occupy positions in between the molecular herringbone layers of pentacene. When interlayer doping is assumed, there is only place for one $\mathrm{K}$ atom per organic molecule because larger amounts of potassium imply too small distances among dopants. Actually, the impossibility of having doubly negatively charged pentacene ions was remarked in Sec. IV of Ref. [14]. However, subsequent experimental works did not recover a Mott-Hubbard transition as a function of potassium doping but a constant insulator ground state. Bussolotti et al. used angleresolved ultraviolet photoemission spectroscopy to reach this conclusion by doping of single crystalline pentacene thin films grown on $\mathrm{Cu}(110)$ [15]. Roth and Knupfer presented electron energy-loss spectroscopy results for potassium doped samples of tetracene and pentacene that were characterized by a finite energy gap in the excitation spectra, i.e., none of the samples became metallic [16]. In the last work, a maximum $\mathrm{K}$ doping of two atoms of potassium per pentacene molecule was achieved. 
Potassium intercalation of pentacene remains unclear until the present moment. Two papers published in 2016 present two quite contradictory scenarios. Phan et al. [17] report on thin films with the 1:1 stoichiometry. Napthalene, anthracene, tetracene, and pentacene are potassium intercalated and all of them show insulating character although the underlying mechanisms are different for short versus long PAHs. On the contrary, Nakagawa et al. [18] analyze the properties of a wide range of $\mathrm{K}$ intercalated pentacene samples and observe superconductivity for a large 3:1 stoichiometry. The crystalline structure of the superconducting phase is no longer triclinic but monoclinic.

Definitely, potassium intercalation of pentacene calls for some clarification. Most probably, important differences must be associated to structural differences of samples that are synthesized in different ways. Therefore, theoretical band structure calculations based on well defined crystalline structures can help to understand different possible scenarios. Moreover, although the prediction capabilities of density functional theory (DFT) are not absolute, the possibility of comparing several structures analyzed with exactly the same approach provides important advantages. In our work, we have systematically investigated all the suggested crystalline structures of potassium intercalated pentacene emphasizing low doping level (just one $\mathrm{K}$ atom per pentacene molecule) and the greatest plausible doping level (three $\mathrm{K}$ atoms per pentacene molecule). The possible appearance of magnetic instabilities that can drive the compound to an insulating phase have been taken into account in our investigation. In this way, a set of well converged crystalline phases that bear all the structural motives described so far in the literature has been produced and sorted by their final cell energies. They have been collected as cif files in the Supplemental Material [19]. In particular, an outstanding new phase has been obtained for $\mathrm{K}_{3}$ Pentacene that shows a monoclinic cell as found in Ref. [18] but structurally quite different from the model proposed in Fig. 6 of this reference. Our band structure calculation predicts metallic behavior for the new crystalline structure.

The rest of the paper is organized as follows. Section II is devoted to give some details of the methods and procedures used in this work. Section III presents our main computational results together with some discussion of them. The work ends with a few final concluding remarks (Sec. IV).

\section{COMPUTATIONAL PROCEDURES}

Theoretical calculations for model systems of interest have been performed using ab initio van der Waals-density functional theory (vdW-DFT) as proposed in 2004 by Dion et al. [20]. Taking advantage of an algorithm introduced by Román-Peréz and Soler the whole calculation can be done in reciprocal space [21]. Actually, we have used a later development of this approximation [22] that was coded by J. Klimeš within the VASP program [23-26]. Wave functions have been expanded in a plane-wave basis set up to a cutoff of $700 \mathrm{eV}$ and sampled on a $\Gamma$ centered Monkhorst-Pack grid automatically generated taking into account the sizes of the reciprocal lattice vectors (about fifty points during geometry optimizations and about eight hundred to get precise densities of states using the tetrahedra method). Core electrons have
TABLE I. Main properties of the studied K doped pentacene structures. Volume, spin polarization, and total energy are given per cell. The corresponding cif files are given as Supplemental Material ([19]).

\begin{tabular}{lcccr}
\hline \hline Compound & Structure & Volume $\left(\AA^{3}\right)$ & Pol. (e) & Energy (eV) \\
\hline Potassium & bcc & 68.79 & 0 & 1.045 \\
Pentacene & LT & 688.67 & 0 & -435.542 \\
& HT & 688.07 & 0 & -435.539 \\
& intralayer & 801.57 & 0 & -435.416 \\
& intralayer & 801.57 & 2.007 & -435.505 \\
KPentacene & interlayer & 728.89 & 0 & -435.169 \\
& interlayer & 728.89 & 0.032 & -435.169 \\
& dimerized & 791.20 & 0 & -435.634 \\
& dimerized & 791.20 & 2.006 & -435.718 \\
$\mathrm{~K}_{2}$ Pentacene & herringbone & 816.26 & 0 & -436.449 \\
& herringbone & 831.23 & 0 & -434.822 \\
$\mathrm{~K}_{3}$ Pentacene & herringbone & 831.23 & 2.007 & -434.874 \\
& monoclinic & 862.44 & 0 & -434.054 \\
& monoclinic & 862.44 & 1.060 & -434.053 \\
\hline \hline
\end{tabular}

been treated within the projector augmented method [27,28]. Semicore $3 \mathrm{~s}$ and $3 \mathrm{p}$ potassium states have been considered valence states, i.e., they have been fully self-consistently included. The relaxation of the electronic degrees of freedom has been stopped when both the total energy and the band structure energy variations between two steps are smaller than $10^{-4} \mathrm{eV}$. Ionic relaxation has been continued as long as any force were larger than $0.01 \mathrm{eV} / \AA$.

Our whole procedure gives very satisfactory results when it is confronted with two realistic tests. Firstly, polymorphism in pristine pentacene has been studied by calculating the two polymorphs described for bulk crystalline pentacene [29]. Calculations starting from pentacene-HT and pentacene-LT experimental structures afford the corresponding minima with interlayer spacing of $d(001)=14.4$ and $14.1 \AA$, respectively. Optimized structures closely resemble the experimental ones [30]. Meanwhile, the energy difference between both structures is as small as $3 \mathrm{meV}$ (see Table I).

Secondly, the structure of the $\mathrm{KC}_{8}$ intercalation compound has been simulated [31]. In this case the behavior of potassium as an intercalation element between carbon aromatic structures is checked. Theory gives an interplanar distance of $5.48 \AA$ that compares satisfactorily with the experimental value of $5.35 \AA$. A more accurate comparison can be made using the corresponding cif files. We have used VASP Data Viewer to represent isosurfaces of the electronic density in crystals and wxMacMolPlt to visualize molecular orbitals of an isolated pentacene molecule [32].

\section{RESULTS AND DISCUSSION}

\section{A. KPentacene compound}

It is usually believed that when tiny amounts of potassium are introduced into a pure crystalline sample of pentacene, the original band structure is not modified but simply populated by the outer $4 \mathrm{~s}$ electrons of potassium. The upper (a) panel of Fig. 1 shows the density of states of pristine pentacene at 


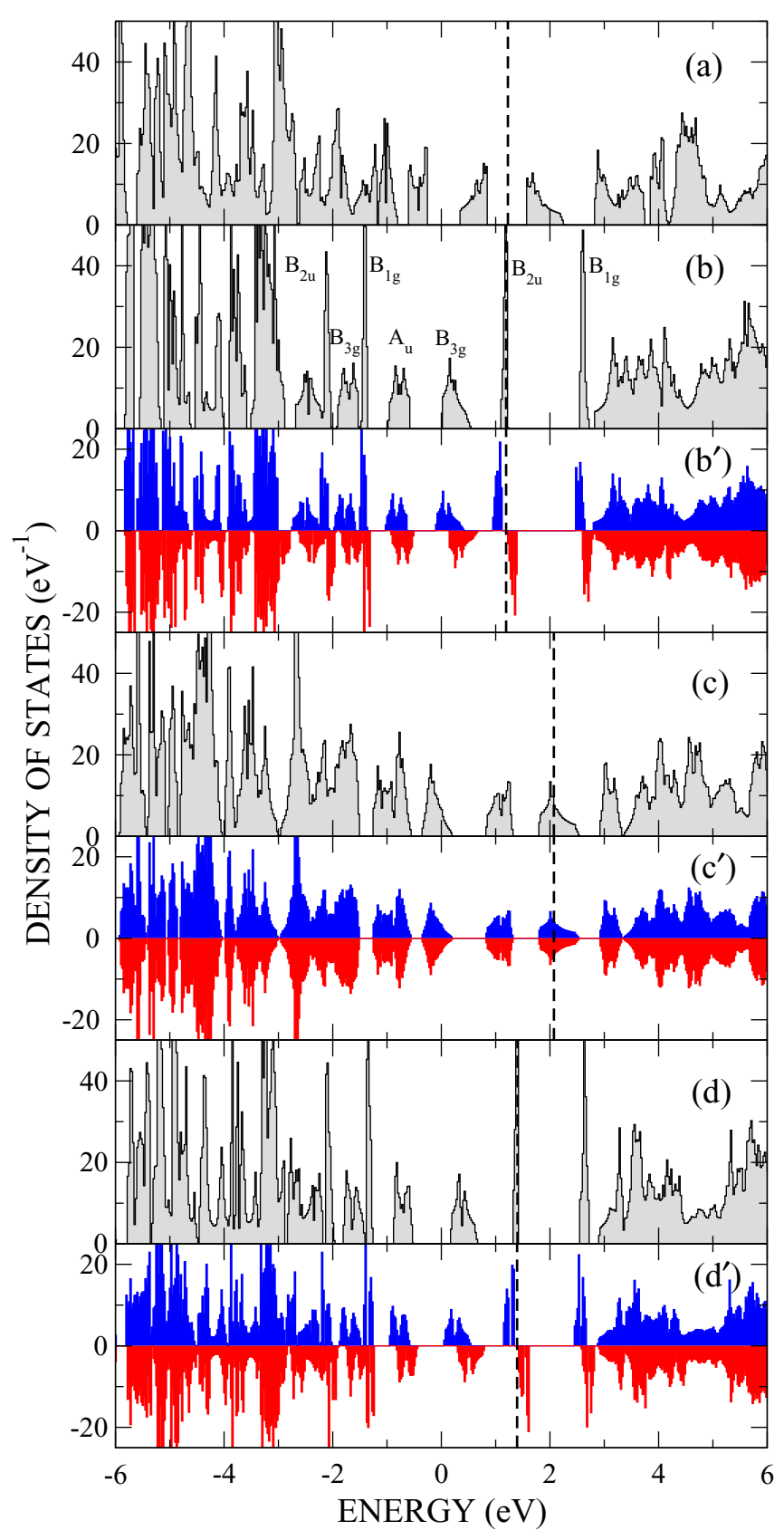

FIG. 1. From top to bottom, (a) density of states (DOS) of pristine pentacene, (b) DOS for monopotassium pentacene (herringbone structure, intralayer doping), ( $b^{\prime}$ ) same but allowing spin polarization (majority spin polarization positive and blue colored, minority spin polarization negative and red colored), (c) DOS for monopotassium pentacene for potassium atoms located between pentacene layers (interlayer doping) and ( $\left.\mathrm{c}^{\prime}\right)$ same but allowing spin polarization, and finally, (d) DOS for monopotassium pentacene doubling the unit cell to allow the formation of pentacene dimers bound by two potassium atoms and $\left(\mathrm{d}^{\prime}\right)$ spin polarized result. The second panel (b) shows our assignment of some peaks of the DOS to molecular orbitals of pentacene isolated molecule. Fermi levels are indicated by black dashed lines.

the optimized theoretical structure that practically coincides with the experimentally determined structure at low temperature [33]. Two not so narrow bands define the semiconducting gap. As long as this scenario is valid, the actual positions of dopants are irrelevant since the electric potential due to potassium cations is not taken into account. In this case, metallic electronic transport would be possible as long as many-body effects in the form of a large Hubbard $U$ were absent. Results given in Refs. [10,11,14] could be understood in this context. On the other hand, additional experimental results point to a prompt metal-insulator transition at the first stages of doping [15-17]. A precise description of pentacene compounds with less than one potassium atom per unit cell could be theoretically done using crystal supercells of pentacene crystal. We have not aimed at that goal in this work. Therefore, we start calculations for a 1:1 K:Pentacene stoichiometry.

If the layers of pentacene showing the herringbone pattern were compact enough, potassium atoms would occupy interlayer positions. In this way, typical $\mathrm{K}-\mathrm{K}$ distances are somewhat larger than $4.5 \AA$ for a KPentacene compound, that is, quite similar to the first-neighbor distance in pure crystalline potassium. Consequently, further doping would be either impossible or giving place to interlayer metallic potassium (two or more $\mathrm{K}$ planes). All these inconveniences are avoided if dopant atoms penetrate into the herringbone layer producing an intralayer compound (see upper panel of Fig. 2). This is certainly the case for picene since doping as high as 3:1 has been reached. Theoretical estimation of free energies for both structural possibilities strongly point to intralayer doping. Numerical results compiled in Table I show that intralayer doping is about $0.7 \mathrm{eV}$ per cell more stable than interlayer doping. Stabilization is still larger when spin polarized solutions are considered. Final structures are given as cif files in the Supplemental Material [19]. Later we will discuss how spin polarization appears and how it strongly suggests isolating behavior for this compound.

We have also considered the formation of pentacene dimers with two potassium atoms between hydrocarbon molecules as suggested by Ref. [17]. To this end we start forming an isolated $\mathrm{K}_{2}$ Pentacene 2 dimer that is later used as the unit of a herringbone structure [34]. After a long and cumbersome geometry optimization, a conventional herringbone structure is recovered (see lower panel of Fig. 2). Nevertheless, this lattice shows one important difference when compared with the previous one since now potassium atoms are grouped in pairs. Actually, it is still possible to look at this structure as a herringbone array of pentacene- $\mathrm{K}_{2}$-pentacene complexes. Corresponding cell energies are given in Table I. They indicate that in spite of the reduced homogeneity this crystalline structure provides the absolute minimum at a 1:1 stoichiometry by $\approx 0.2 \mathrm{eV}$ per cell. This dimerized KPentacene structure could be used as a starting model for a $\mathrm{K}_{0.5}$ Pentacene compound (only one $\mathrm{K}$ atom per cell) substituting $\mathrm{K}$ pairs by isolated atoms or also for a $\mathrm{K}_{1.5}$ Pentacene compound if three instead of two $\mathrm{K}$ atoms occupy half of the channels of the herringbone structure. In any case, going to $\mathrm{K}$ richer compounds is not possible since $\mathrm{K}$ groups formed by four or more atoms are not stable.

Searching for additional possible crystalline structures of KPentacene, we have also employed the structures obtained by Hansson et al. as unbiased seeds [13]. The resulting converged structures does not differ very much from the initial ones. However, the calculated total energies are well above the values 

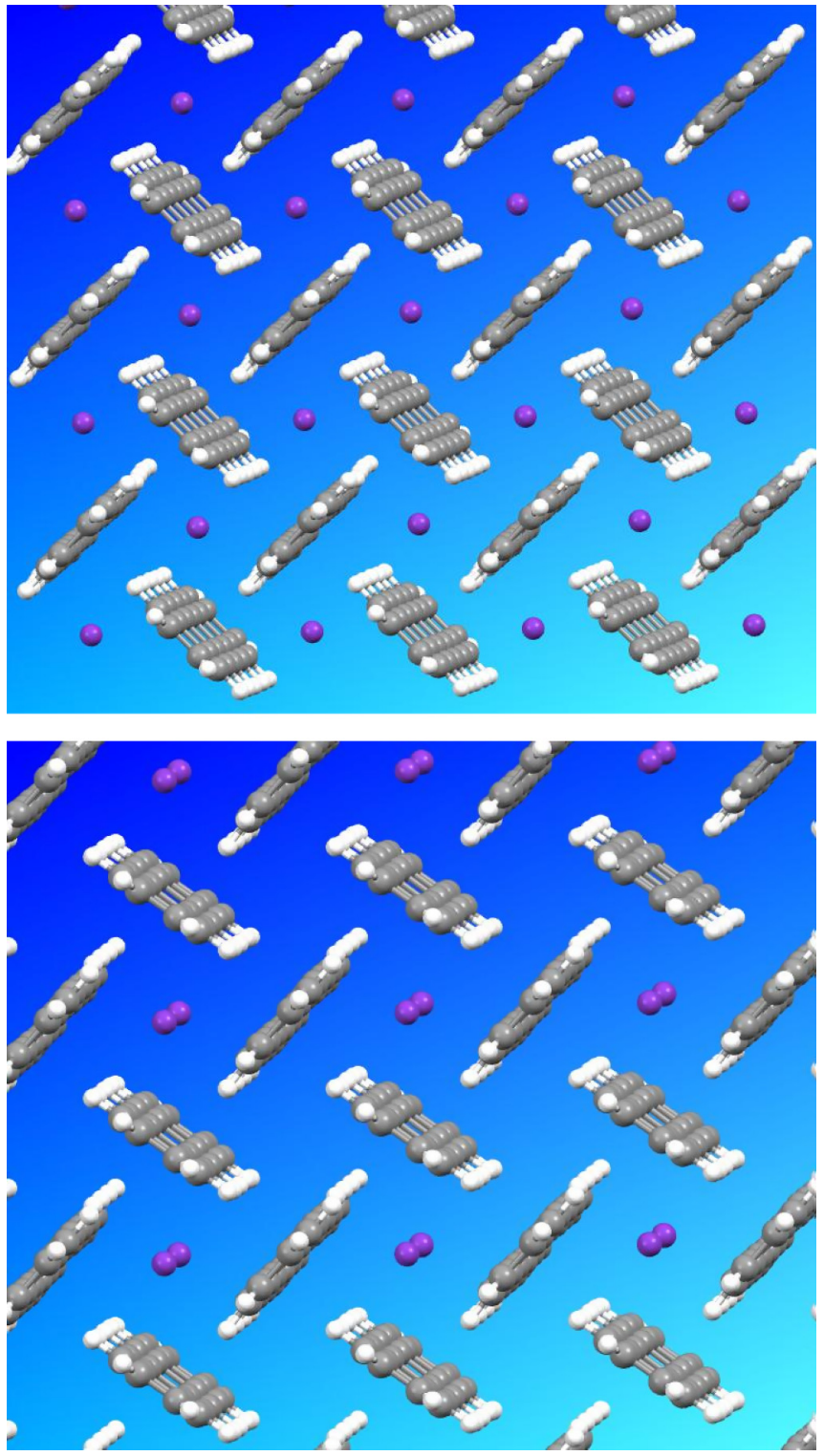

FIG. 2. Herringbone structure of KPentacene (top) compared to the converged crystalline structure obtained starting from $\mathrm{K}_{2}$ Pentacene ${ }_{2}$ dimers (bottom).

of the structures pictured in Fig. 2 and, consequently, they have not been further analyzed.

In order to understand electronic transport and magnetic properties of KPentacene, we have calculated detailed densities of states (DOS) for the relevant structures. Figure 1 shows how the change of the bands after doping strongly depend on the precise position of $\mathrm{K}$ dopants. Upper panel shows the DOS of pristine pentacene. Two not so narrow bands define the semiconducting gap. The second panel gives the DOS for the more stable intralayer structure. The important narrowing of several bands is quite noticeable. It is due to the symmetry of the corresponding pentacene molecular orbitals. After structural optimization, pentacene molecules show parallel long axis and almost perpendicular molecule planes. Molecular orbitals are antisymmetric relative to the molecule plane since they are linear combinations

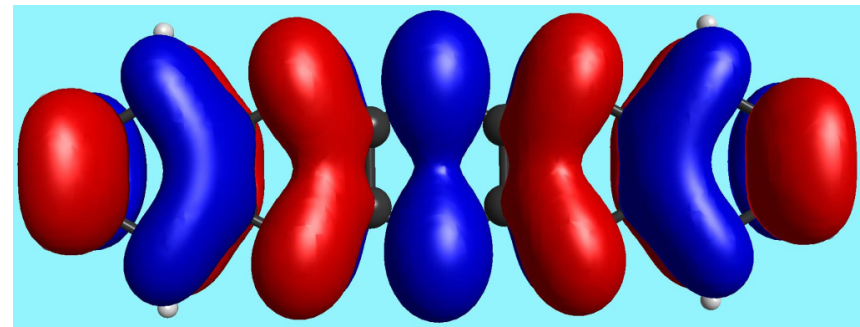

FIG. 3. Picture of LUMO of the pentacene molecule $\left(B_{2 u}\right.$ symmetry).

of $\pi$ orbitals. Therefore, they only overlap with molecular orbitals of neighboring molecules that are also antisymmetric relative to the symmetry plane that is perpendicular to the molecule and contains its long axis. This is not the case for lowest unoccupied molecular orbital (LUMO) and LUMO+1 that belong, respectively, to $\mathrm{B}_{2 \mathrm{u}}$ and $\mathrm{B}_{1 \mathrm{~g}}$ representations of the molecule point symmetry group $\mathrm{D}_{2 \mathrm{~h}}$. Pictures of them are given in Figs. 3 and 4. This property allows an easy assignment of the most relevant bands to specific molecular states. Since Fermi level divides the $B_{2 u}$ band into occupied and unoccupied parts one could naively think that the compound would show a metallic behavior. Nothing is further from reality, because electron interaction leads to spin polarized bands that are completely occupied and completely empty, respectively. Although our calculation shows a ferromagnetic solution, i.e., spins of the two doping electrons are parallel, we think that different spin configurations could compete (for example, antiparallel spins within the cell). Nevertheless, the relevant point is that correlation opens a gap in the DOS. In conclusion, structural changes following doping invalidate a naive picture of electrons populating a relatively broad band (upper panel of Fig. 1 corresponding to pristine pentacene).

This scenario could be completely different if interlayer doping were possible. As shown in fourth (c) and fifth $\left(\mathrm{c}^{\prime}\right)$ panel of Fig. 1, DOS approximately resembles the undoped bands in this case. The possibility of spin polarization is marginal for this structural model (see Table I). The position of Fermi level points to a conducting behavior. Although this

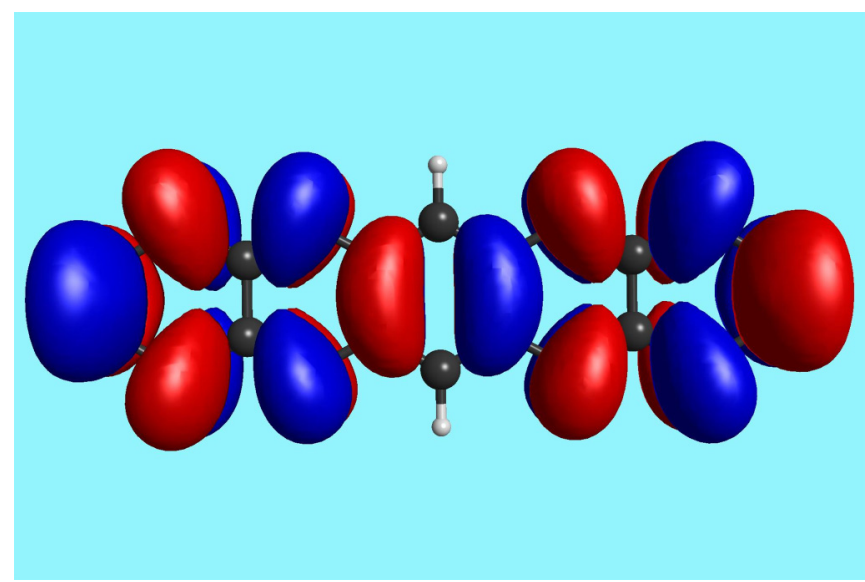

FIG. 4. Picture of LUMO+1 of the pentacene molecule $\left(\mathrm{B}_{1 \mathrm{~g}}\right.$ symmetry). 
structure is energetically unlikely, perhaps the detailed way in which samples are grown matters and could eventually explain metallic behavior $[10,11,14]$. In any case, as said previously, interlayer doping is limited to one electron ( $\mathrm{K}$ atom) per pentacene molecule. Further dopants either form metallic K layers or go into the pentacene herringbone layer.

Bottom (d) and (d') panels of Fig. 1 give the density of states calculated for the dimerized structure. The second one corresponds to the spin polarized solution involving $\approx 2$ more electrons with up spin than with down spin in every cell. Energy gain is modest, $\approx 0.08 \mathrm{eV}$ per cell. Both figures are very similar to the values obtained for the more homogeneous solution shown in (b) and ( $\left.b^{\prime}\right)$ panels of Fig. 1. In fact, the whole DOS is very similar. This is not a surprise since in both cases LUMO and LUMO +1 states of pentacene describe the relevant bands, being the influence of the precise position of $\mathrm{K}$ cations not so important. Nevertheless, magnetic DOS shows additional small splittings in the dimerized case. Let us comment here that the insulating character of our solution does not come for some kind of Peirls deformation but for large correlation effects associated with the very narrow half-filled band. This makes a difference with arguments found in Ref. [17].

\section{B. $\mathbf{K}_{2}$ Pentacene compound}

It is easy to stabilize pairs of $\mathrm{K}$ atoms within the herringbone structure of pentacene. It looks like the lower panel of Fig. 2 once all channels are filled with pairs of dopants. Complete structural data are given as Supplemental Material [19]. Main characteristics are given in Table I. The corresponding DOS looks like panel (b) of Fig. 1 but now the Fermi level is located between $B_{2 u}$ and $B_{1 g}$ peaks. This stoichiometry provides maximum lattice stability but not metallic properties. Unfortunately, this fact considerably reduces its potential use and we have not continued its study.

\section{C. $\mathbf{K}_{3}$ Pentacene compound}

One of the main advantages of our favorite intralayer doping is that it easily allows additional dopants. For example, three instead of one or two $\mathrm{K}$ atoms can be accommodated within the empty regions of the herringbone structure. Complete structural data are provided by P-1.cif file of Supplemental Material [19]. Top panel of Fig. 5 shows the arrangement of one of these herringbone layers. Notice that when dopants enter this way into the herringbone structure, the spatial crystal symmetry is preserved, that is, a triclinic $P_{-1}$ space group characterizes the compound as it already happens for pristine pentacene. Nevertheless, very recent results reported by Nakagawa et al. [18] for potassium intercalated pentacene show important structural and electronic changes for the larger 3:1 stoichiometry. The crystal undergoes a phase transition from the triclinic structure to a new monoclinic one. Drastically changing the initial crystal structure of a $\mathrm{K}_{3}$ Pentacene compound, we have achieved the structural stabilization of a brand new phase in which former herringbone structure reassembles completely leaving layers of slipped parallel pentacene molecules. A complete characterization of the crystalline structure of this monoclinic phase is given by C2m.cif file [19]. Besides, bottom panel of Fig. 5 allows a
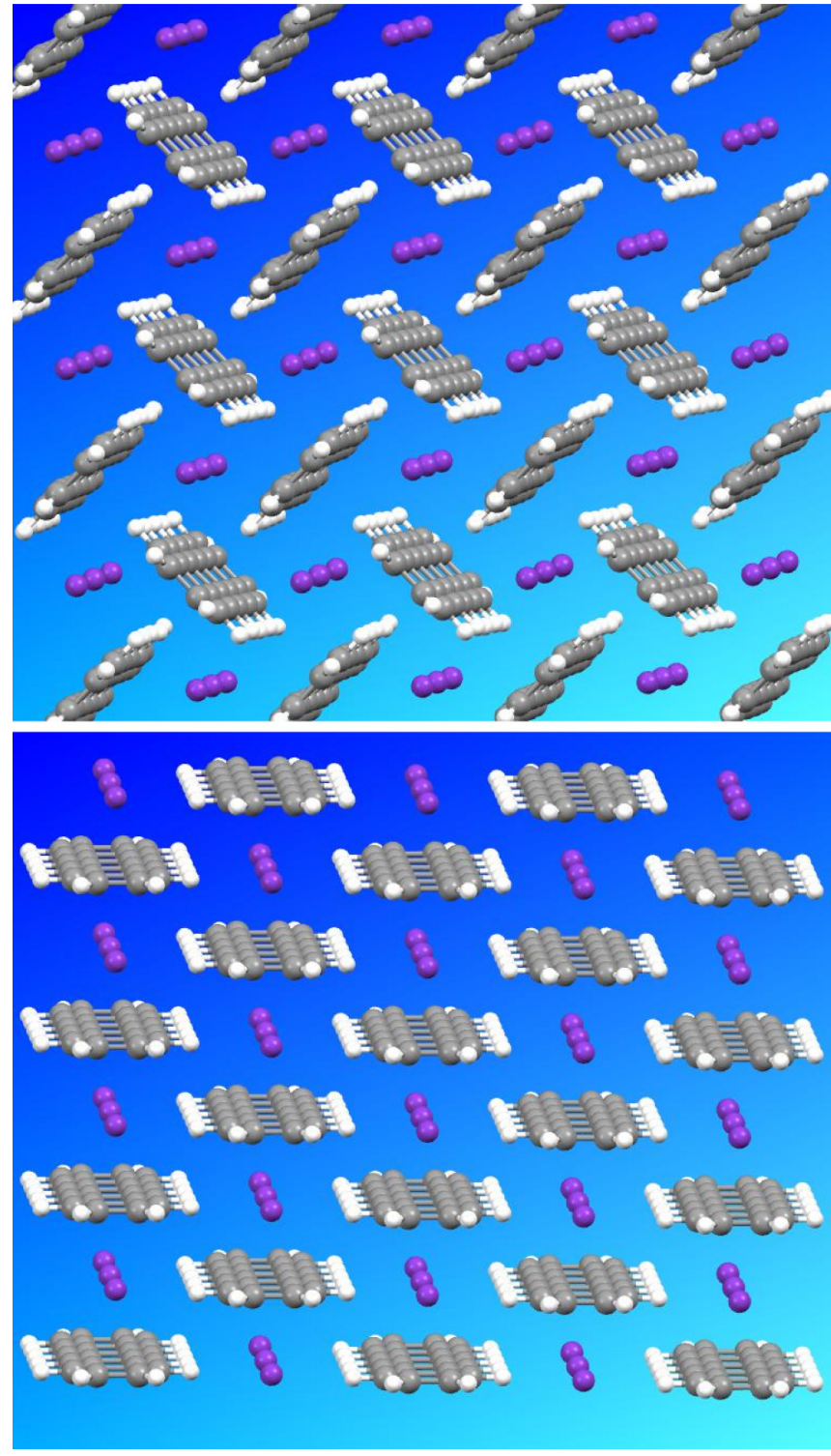

FIG. 5. Herringbone structure of $\mathrm{K}_{3}$ Pentacene (top) compared to the new monoclinic one (bottom).

visual first impression of the important structural changes. Layers show a kind of rectangle centered structure which can be considered typical of an ionic compound. While lattice parameters of the herringbone phase are not so different from the parameters of the pristine structure, changes needed to arrive to the monoclinic lattice are very important (see Table II). Agreement with experimentally determined lattice parameters is reasonable (see data in Table II of Ref. [18]). Perhaps the most noticeable change is the perfect parallel arrangement of pentacene molecules. It allows larger hopping values among pentacene LUMO (LUMO+1) states and, therefore, wider half-filled bands that should allow metallic electronic transport properties. Summarizing, our numeric search provides two quite different crystalline structures for $\mathrm{K}_{3}$ Pentacene in agreement with recent experimental findings [18]. Although the new monoclinic phase is energetically unfavorable (see data in Table I), detailed kinetic processes could produce this very regular structure. 
TABLE II. Lattice parameters for pentacene (PA) and K-doped pentacene. Spatial groups are indicated for each structure.

\begin{tabular}{lcccc}
\hline \hline & $\mathrm{PA}\left(P_{-1}\right)$ & $\mathrm{KPA}\left(P_{-1}\right)$ & $\mathrm{K}_{3} \mathrm{PA}\left(P_{-1}\right)$ & $\mathrm{K}_{3} \mathrm{PA}\left(C_{2 / m}\right)$ \\
\hline$a(\AA)$ & 6.26 & 7.64 & 7.62 & 10.63 \\
$b(\AA)$ & 7.84 & 7.70 & 7.76 & 5.50 \\
$c(\AA)$ & 14.52 & 14.02 & 14.37 & 14.88 \\
$\alpha\left({ }^{\circ}\right)$ & 76.2 & 90.9 & 91.9 & 90.0 \\
$\beta\left(\left(^{\circ}\right)\right.$ & 88.0 & 102.8 & 102.0 & 97.5 \\
$\gamma\left({ }^{\circ}\right)$ & 84.7 & 95.2 & 94.8 & 90.0 \\
\hline \hline
\end{tabular}

Let's discuss relevant differences in the corresponding band structures. DOS for both of them are shown in Fig. 6. Band structure of $\mathrm{K}_{3}$ Pentacene in the herringbone structure closely resembles the result obtained for smaller doping in a similar structure. Occupation is the main difference. Bands are assigned to particular molecular orbitals of pentacene in the same way. As it happens for smaller doping, relevant bands

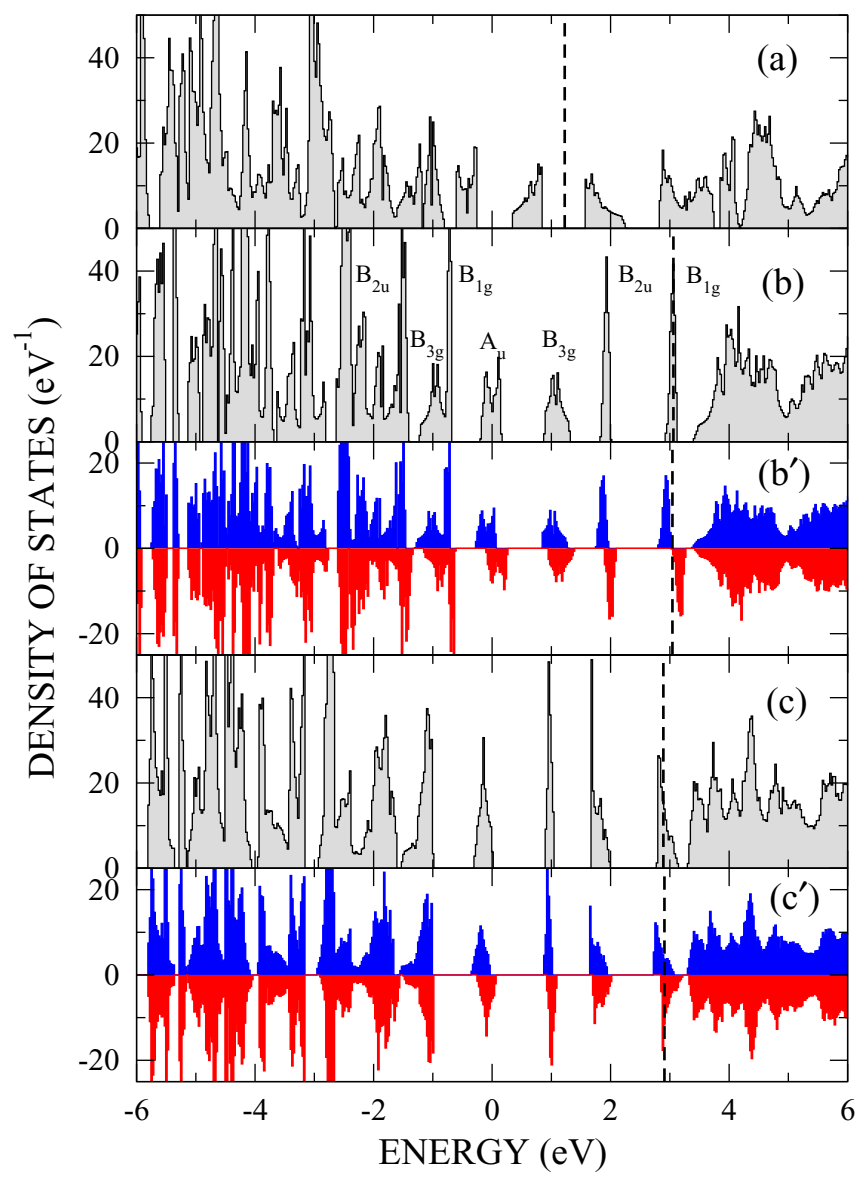

FIG. 6. From top to bottom, (a) density of states (DOS) of pristine pentacene, (b) DOS for tripotassium pentacene (herringbone structure), ( $\left.b^{\prime}\right)$ same but allowing spin polarization (majority spin polarization positive and blue colored, minority spin polarization negative and red colored), (c) DOS for tripotassium pentacene in the new monoclinic structure and, $\left(\mathrm{c}^{\prime}\right)$ same but allowing spin polarization. The assignment of some peaks to molecular orbitals of pentacene molecules follows the analysis done for Fig. 1. Black dashed lines indicate Fermi level positions. are quite narrow. Therefore, magnetic instabilities should be expected. Actually, both $\mathrm{B}_{2 \mathrm{u}}$ and $\mathrm{B}_{1 \mathrm{~g}}$ polarize completely to gain about $0.05 \mathrm{eV}$ per cell. As it happened at lower doping, we get a ferromagnetic solution due to the initial polarization conditions. An antiferromagnetic solution showing opposite spin polarization in the two pentacene molecules forming the unit cell is quite possible although the expected energy change would be not significant at our computational accuracy level. Old results by Mori and Ikehata [11] have predicted magnetic phases at low temperatures. The appearance of spin polarization has also been investigated for the new monoclinic phase. In this case a ferromagnetic solution is still possible but energetically unlikely. Table I compiles energies and total cell spin polarization for both structures. At this point is it possible to predict metallicity for the monoclinic structure. While the herringbone structure shows a robust spin polarization giving rise to an insulating DOS $\left[(b)\right.$ and $\left(b^{\prime}\right)$ panels of Fig. 6], the smaller value of spin polarization allows a clear metallic Fermi level in the monoclinic phase. Actually, the broadening of the $\mathrm{B}_{1 \mathrm{~g}}$ band comes from a not very large $\pi-\pi$ overlap between LUMO +1 states of slipped parallel pentacene molecules. Although it is considerably larger than for the herringbone structure, it is not so large as to definitely discard any many-body instability.

Densities of charge corresponding to the electrons donated by $\mathrm{K}$ have been plotted for the most stable herringbone structure. Figures 7 and 8 give isosurfaces than can nicely be interpreted as corresponding to LUMO and LUMO+1

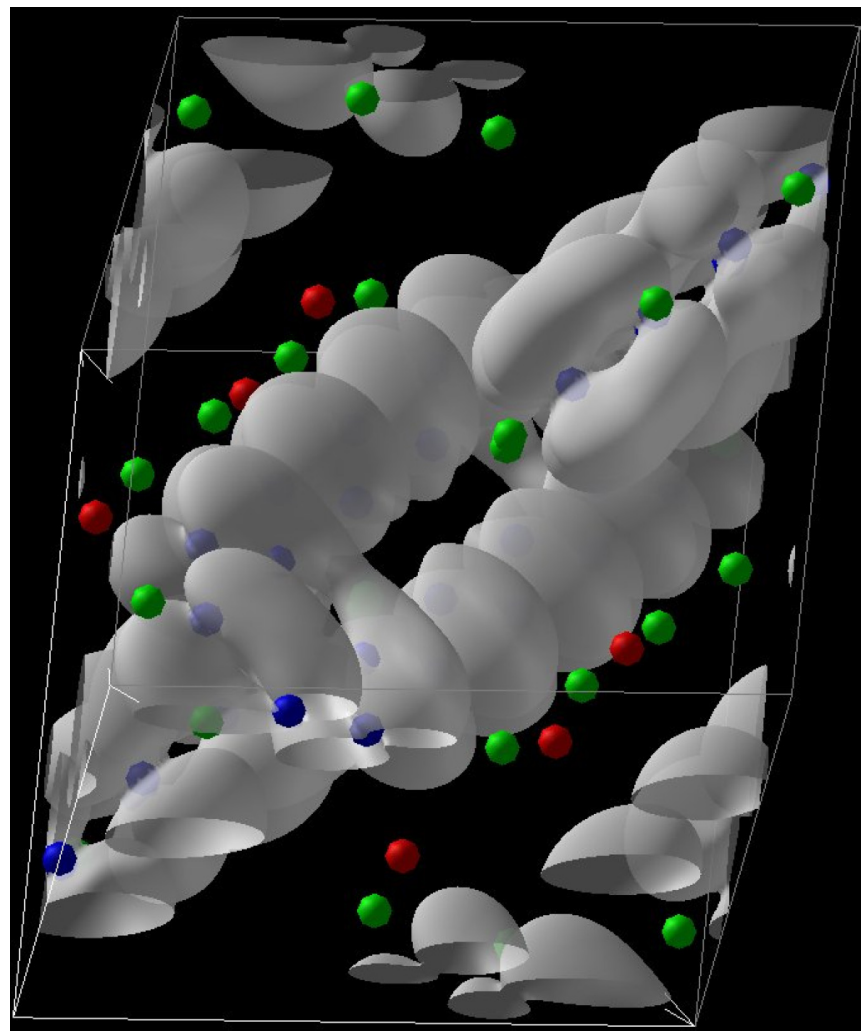

FIG. 7. Isosurface corresponding to the charge density of $\mathrm{K}_{3}$ Pentacene electrons in bands 127 and $128\left(\mathrm{~B}_{2 \mathrm{u}}\right)$, i.e., the first four $\mathrm{K}$ electrons donated to pentacene (isosurface value is 6). 


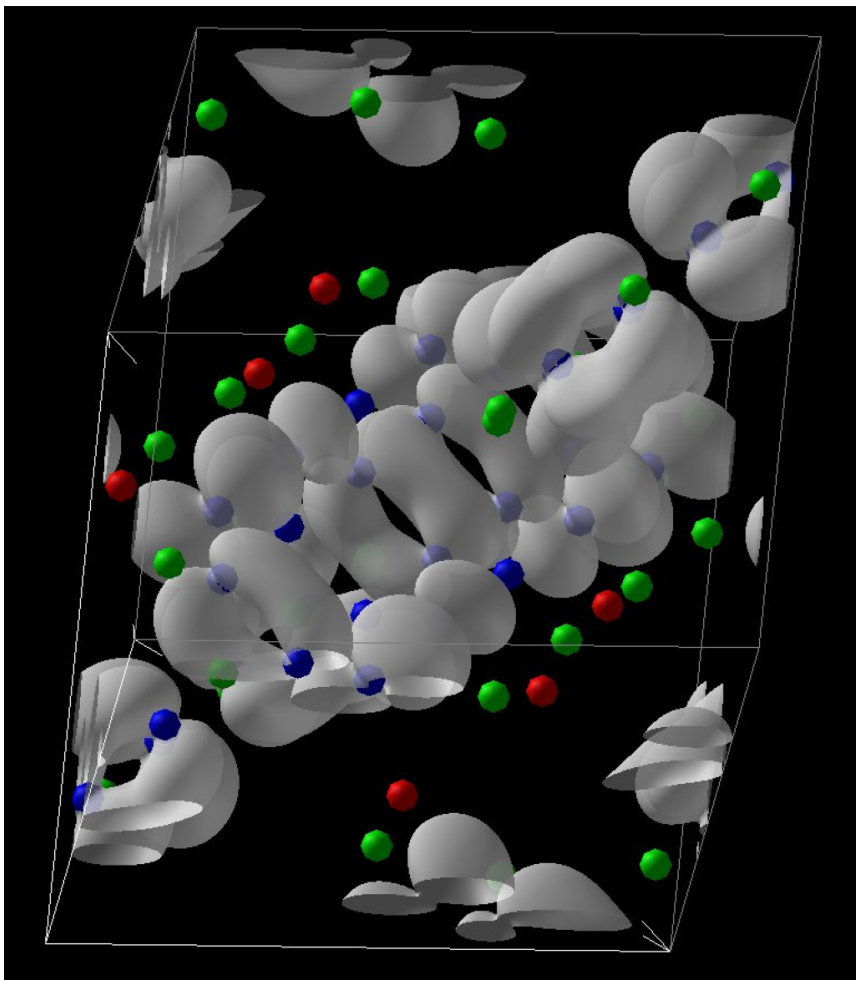

FIG. 8. Isosurface corresponding to the charge density of $\mathrm{K}_{3}$ Pentacene electrons in bands 129 and $130\left(\mathrm{~B}_{1 \mathrm{~g}}\right)$, i.e., the last two $\mathrm{K}$ electrons donated to pentacene (isosurface value is 6).

pentacene states. Therefore our previous assignation of doping bands to particular molecular orbitals is confirmed. A similar result has been obtained for the monoclinic structure.

An estimation of the powder patterns corresponding to the two structures stabilized in our work can be easily obtained from the given structural cif files. Unfortunately, a detailed comparison with experimentally collected data is not possible without a reinterpretation of data based on the new proposed theoretical model.

\section{Formation energies}

Formation energies of the $\mathrm{K}_{x}$ Pentacene phases studied in our work can be calculated using the data given in Table I. For example, the formation energy of $\mathrm{K}_{2}$ Pentacene from metallic potassium and pure pentacene is obtained by:

$$
E\left(\mathrm{~K}_{2} \text { Pentacene }\right)-E(\text { Pentacene })-4 E(\mathrm{~K}),
$$

giving $-5.09 \mathrm{eV}$ per cell whereas the formation energy of the
TABLE III. Formation energies preserving the herringbone structure. Data from Table I have been used. Energies are given in $\mathrm{eV}$ per crystal cell. The second column gives formation energies relative to the previous compound in order to emphasize relative stabilities.

\begin{tabular}{lll}
\hline \hline KPentacene & -1.96 & -1.96 \\
$\mathrm{~K}_{2}$ Pentacene & -5.09 & -3.12 \\
$\mathrm{~K}_{3}$ Pentacene & -5.55 & -0.46 \\
\hline \hline
\end{tabular}

same compound by the addition of potassium to KPentacene is given by:

$$
E\left(\mathrm{~K}_{2} \text { Pentacene }\right)-E(\text { KPentacene })-2 E(\mathrm{~K}),
$$

resulting in $-3.12 \mathrm{eV}$ per cell. Table III compiles the values corresponding to the $\mathrm{K}_{x}$ Pentacene phases preserving the herringbone structure. All compounds are stable although $\mathrm{K}_{2}$ Pentacene shows a preferred stability as the second column of the Table points out. Actually, the separation of KPentacene crystal into pure potassium and $\mathrm{K}_{2}$ Pentacene phases involves an energy gain of $+0.58 \mathrm{eV}$ per cell.

\section{CONCLUDING REMARKS}

Stable structural phases of K-doped pentacene have been theoretically analyzed using a precise computational scheme. From the point of view of thermodynamic stability, potassium does always enter into the herringbone structure of the pristine pentacene crystal in an intralayer manner. Other structural alternatives like interlayer doping produce higher values of the cell energy. Extra electrons populate pentacene LUMO firstly and pentacene LUMO+1 afterward. Simultaneously, they lead to a subtle molecular rearrangement of the herringbone structure that maximizes symmetry and produces very narrow bands. Therefore, important correlation effects have to be expected that open the door to either magnetic insulating phases as shown in our work or more exotic solutions. On the other hand, kinetically driven reaction pathways may make possible the stabilization of a new monoclinic structure showing about $0.77 \mathrm{eV}$ higher energy per cell. If this happened, our results point to a metallic band structure for this crystalline phase. In any case, a detailed experimental determination of the structure of doped pentacene is needed before closing the quest for a metal based on this PAH.

\section{ACKNOWLEDGMENTS}

Financial support by the Spanish Ministry of Economy and Competitiveness (Grants No. FIS2015-64222-C2-1-P and No. MAT2016-78625-C2-2-P) and the University of Alicante is gratefully acknowledged.
[1] R. Mitsuhashi, Y. Suzuki, Y. Yamanari, H. Mitamura, T. Kambe, N. Ikeda, H. Okamoto, A. Fujiwara, M. Yamaji, N. Kawasaki, Y. Maniwa, and Y. Kubozono, Nature (London) 464, 76 (2010).

[2] K. Teranishi, X. He, Y. Sakai, M. Izumi, H. Goto, R. Eguchi, Y. Takabayashi, T. Kambe, and Y. Kubozono, Phys. Rev. B 87, 060505(R) (2013).
[3] Y. Kubozono, R. Eguchi, H. Goto, S. Hamao, T. Kambe, T. Terao, S. Nishiyama, L. Zheng, X. Miao, and H. Okamoto, J. Phys.: Condens. Matter 28, 334001 (2016).

[4] T. Kosugi, T. Miyake, S. Ishibashi, R. Arita, and H. Aoki, J. Phys. Soc. Jpn. 78, 113704 (2009). 
[5] Y. Kubozono, H. Mitamura, X. Lee, X. He, Y. Yamanari, Y. Takahashi, Y. Suzuki, Y. Kaji, R. Eguchi, K. Akaike, T. Kambe, H. Okamoto, A. Fujiwara, T. Kato, T. Kosugi, and H. Aoki, Phys. Chem. Chem. Phys. 13, 16476 (2011).

[6] P. L. de Andres, A. Guijarro, and J. A. Vergés, Phys. Rev. B 83, 245113 (2011).

[7] G. Giovannetti and M. Capone, Phys. Rev. B 83, 134508 (2011).

[8] T. Kosugi, T. Miyake, S. Ishibashi, R. Arita, and H. Aoki, Phys. Rev. B 84, 214506 (2011).

[9] M. Kim, B. I. Min, G. Lee, H. J. Kwon, Y. M. Rhee, and J. H. Shim, Phys. Rev. B 83, 214510 (2011).

[10] T. Minakata, M. Ozaki, and H. Imai, J. Appl. Phys. 74, 1079 (1993).

[11] T. Mori and S. Ikehata, J. Appl. Phys. 82, 5670 (1997).

[12] All three lattice constants are larger in the doped sample although the increase of $c$ is ten times larger than $a$ and $b$ increases. The discussion of the authors is somewhat ambiguous; doping is roughly between pentacene layers but some amount goes intralayer.

[13] A. Hansson, J. Böhlin, and S. Stafström, Phys. Rev. B 73, 184114 (2006).

[14] M. F. Craciun, G. Giovannetti, S. Rogge, G. Brocks, A. F. Morpurgo, and J. van den Brink, Phys. Rev. B 79, 125116 (2009).

[15] F. Bussolotti, S. Kera, and N. Ueno, Phys. Rev. B 86, 155120 (2012).

[16] F. Roth and M. Knupfer, J. Chem. Phys. 143, 154708 (2015)

[17] Q. T. N. Phan, S. Heguri, H. Tamura, T. Nakano, Y. Nozue, and K. Tanigaki, Phys. Rev. B 93, 075130 (2016).

[18] T. Nakagawa, Z. Yuan, J. Zhang, K. V. Yusenko, C. Drathen, Q. Liu, S. Margadonna, and C. Jin, J. Phys.: Condens. Matter 28, 484001 (2016).

[19] See Supplemental Material at http://link.aps.org/supplemental/ 10.1103/PhysRevB.95.134112 for a collection of all relevant crystalline structures mentioned in the main text. They are given in Crystallographic Information File (cif) format as defined by the International Union of Crystallography.

[20] M. Dion, H. Rydberg, E. Schröder, D. C. Langreth, and B. I. Lundqvist, Phys. Rev. Lett. 92, 246401 (2004).

[21] G. Román-Pérez and J. M. Soler, Phys. Rev. Lett. 103, 096102 (2009).

[22] K. Lee, E. D. Murray, L. Kong, B. I. Lundqvist, and D. C. Langreth, Phys. Rev. B 82, 081101 (2010).

[23] J. Klimeš, D. R. Bowler, and A. Michaelides, Phys. Rev. B 83, 195131 (2011).

[24] G. Kresse and J. Hafner, Phys. Rev. B 47, 558 (1993); 49, 14251 (1994).

[25] G. Kresse and J. Furthmüller, Comput. Mater. Sci. 6, 15 (1996).

[26] G. Kresse and J. Furthmüller, Phys. Rev. B 54, 11169 (1996).

[27] P. E. Blöchl, Phys. Rev. B 50, 17953 (1994).

[28] G. Kresse and D. Joubert, Phys. Rev. B 59, 1758 (1999).
[29] These two phases have been referred to as pentaceneHT [also called phase C after Campbell's first report (Ref. [35])] and pentacene-LT [or phase-H after Holmes's first report (Ref. [33])]. A detailed discussion of pentacene polymorphism is given in Refs. [36,37]. Comparatively, the herringbone structure within the layer is virtually identical for both polymorphs, while the interlayer spacing, experimentally identified as $d(001)$, is slightly longer in pentacene-HT $(\approx 14.4 \AA)$ respect to the most common pentacene-LT phase ( $\approx 14.1 \AA$ in average). This experimental distance has been often used to identify each crystallographic phase since their respective unit cell volumes are very similar.

[30] Calculated lattice parameters a, b, c, $\alpha, \beta, \gamma$ are 6.26, 7.84, $14.52,76.2,88.0$, and 84.7 for the low temperature (LT) phase (Ref. [33]) and 6.06, 7.93, 14.76, 97.2, 100.4, and 94.3 for the high temperature (HT) phase (Ref. [35]) whereas experimental values are $6.29,7.81,14.58,76.5,87.6$, and 84.7 for the LT phase (Ref. [38]) and 6.12, 8.08, 14.96, 97.7, 100.2, and 94.0 for the HT phase (Ref. [39]). Distances are given in $\AA$ and angles in degrees. A more detailed comparison can be obtained through the cif files given in the Supplemental Material ([19]).

[31] P. Lagrange, D. Guérard, and A. Hérold, Ann. Chim. (Paris) 3, 143 (1978).

[32] WXMACMOLPLT package has been employed to represent some molecular orbitals of pentacene. It is described by B. M. Bode and M. S. Gordon, J. Mol. Graphics Mod. 16, 133 (1998).

[33] D. Holmes, S. Kumaraswamy, A. J. Matzger, and K. P. C. Vollhardt, Chem. Eur. J. 5, 3399 (1999).

[34] We have stacked the dimers in a herringbone pattern in which the long axis of pentacene molecules is approximately parallel. Nevertheless, Fig. 9 of Ref. [17] shows dimers having a long axis that is almost perpendicular. This stacking is quite different from the original pristine structure. It can be discarded based on energy considerations since our calculations give a difference of about $0.51 \mathrm{eV}$ per doubled cell between both stacking ways.

[35] R. B. Campbell, J. M. Robertson, and J. Trotter, Acta Cryst. 14, 705 (1961).

[36] R. F. della Valle, A. Brillante, E. Venuti, L. Farina, A. Girlando, and M. Masino, Organic Electronics 5, 1 (2004).

[37] T. Siegrist, C. Besnard, S. Haas, M. Schiltz, P. Pattison, D. Chernyshov, B. Batlogg, and C. Kloc, Adv. Mater. 19, 2079 (2007).

[38] Data given by S. Haas, B. Batlogg, C. Besnard, M. Schiltz, C. Kloc, T. Siegrist, P. Pattison, and D. Chernyshov (2006). CCDC 619979: Experimental Crystal Structure Determination; Cambridge Crystallographic Data Centre, http://dx.doi.org/10.5517/ccnt4bx.

[39] Data corresponding to T. Siegrist, C. Besnard, S. Haas, M. Schiltz, P. Pattison, D. Chernyshov, B. Batlogg, and C. Kloc (2006). CCDC 619982: Experimental Crystal Structure Determination; Cambridge Crystallographic Data Centre, http://dx.doi.org/10.5517/cent4f0 after reducing the cell using the Bilbao Crystallographic Server http://www.cryst.ehu.es. 